Case Report

\title{
Cycloserine Induced-Psychosis in a 22-Year Old Male Pharmacy Student: A Case Report
}

\author{
Nkporbu Aborlo Kennedy ${ }^{1, ~ *, ~ A y o d e j i ~ O l u w a s e u n ", ~ A l a s i a ~ D a t o n y e ~ D e n i s ~}{ }^{2}$, \\ Stanley Princewill Chukwuemeka ${ }^{1}$ \\ ${ }^{1}$ Department of Neuropsychiatry, Both University of Port Harcourt Teaching Hospital, Port Harcourt, Nigeria \\ ${ }^{2}$ Department of Internal Medicine, Both University of Port Harcourt Teaching Hospital, Port Harcourt, Nigeria \\ Email address: \\ nakpigi2008@yahoo.com (N. A. Kennedy)
}

\section{To cite this article:}

Nkporbu Aborlo Kennedy, Ayodeji Oluwaseun, Alasia Datonye Denis, Stanley Princewill Chukwuemeka. Cycloserine Induced-Psychosis in a 22-Year Old Male Pharmacy Student: A Case Report. American Journal of Psychiatry and Neuroscience. Vol. 4, No. 1, 2016, pp. 1-4.

doi: 10.11648/j.ajpn.20160401.11

\begin{abstract}
This is case of cycloserine induced-psychosis in a 22-year old male patient on treatment for multi-drugs resistant tuberculosis (MDRTB) at the Aluu MDRTB centre of UPTH. Tuberculosis is a chronic respiratory infection caused by mycobacterium tuberculosis. Cycloserine is a Category IV antimycobacterial agent used for the treatment of resistant tuberculosis. The overall clinical scenerio forms this case report. The aim of this report was to highlight a case of cycloserine induced-psychosis in a 22-year old male patient on treatment for drug resistant tuberculosis at the Aluu MDRTB centre of UPTH and to further point to the fact that it is reversible. The case report of the patient was retrieved and reviewed together with relevant literatures. A 22-year old male patient who was diagnosed of tuberculosis in December, 2013 andrediagnosed as multidrug resistant tuberculosis about 6 months ago. He was admitted as a referral into the MDRTBcentre at Aluu5 months following the later and commenced on a course of Category IV anti TB regimen including cycloserine at a dose of 75 Omg dly. There was no history of use of psychoactive substances and no family history of mental illnesses. His body weight as at the time of the report was $47 \mathrm{~kg}$. Routine and other investigations including full blood count with differencials, electrolyte, urea and creatinine and urinalysis, thyroid function test done were all within normal ranges. Retroviral screening test was seronegative. Within 1 week of commencing the antituberculosis regimen, patient began to manifest a range of psychotic features unusual abnormal behaviours characterized by irrational talks, seeing of strange images unseen by others in clear conscious. After detailed evaluation and with high index of suspicion, a diagnosis of cycloserine-induced psychosis was entertained. The psychiatrist recommended that the cycloerinebe withheld and patient was commenced on low dose atypical antipsychotic agent. Within 48hours, symptoms began to resolve and within 72 hours there was marked improvement particularly in the psychotic symptoms. A good number of medications like cycloserine have been known to induce psychosis. Clinicians should always apply caution particularly in dosage and assess patients fitness for these group of medications. This will help to prevent precipitation of psychiatric adverse symptoms and guarantee the patient optimal care.
\end{abstract}

Keywords: Cycloserine, Psychosis, MDRTB, UPTH

\section{Introduction}

Before now, mental disorders were largely thought to be due to spiritual forces, however, with the advent of medical research, this thinking has shifted as there are now revelations of biological, psychological, social and environmental causes of mental illnesses. A good example of this is the case ofcycloserine induced-psychosis in a 22-year old male patient on treatment for drug resistant tuberculosis at the Aluu DRTB centre of UPTH. Tuberculosis is a chronic respiratory infection caused by mycobacterium tuberculosis [1, 2]. Cycloserine is a Category IV antimycobacterial agent used for the treatment of resistant tuberculosis. Currently, cycloserineis used in conjunction with other tuberculostatic drugs in the treatment of pulmonary or extrapulmonary tuberculosis when primary agents (isoniazid, rifampin, ethambutol, 
pyrazinamide, streptomycin) have failed. The drug is stable in alkaline solution but is rapidly destroyed when exposed to neutral or acidic $\mathrm{pH}[3]$.

Psychiatric symptoms induced by anti-tuberculosis medications specifically multidrug resistant tuberculosis (MDR-TB) therapies are well known in terms of depression, anxiety, and psychosis [4].

Cycloserine is inbibitory for $M$. tuberculosis in concentrations of 5 to $20 \mathrm{pg} / \mathrm{ml}$ in vitro. There is no cross-resistance between cycloserine and other tuberculostatic agents.

Peak concentrations of cycloserine in plasma are reached 3 to 4 hours after a single dose and are in the range of 20 to 35 $\mu \mathrm{g} / \mathrm{ml}$ in children who receive $20 \mathrm{mg} / \mathrm{kg}$; only small quantities are present after 12 hours. Cycloserineis distributed throughout body fluids and tissues. There is no appreciable blood brain barrier to the drug, and CSF concentrations are approximately the same as those in plasma. About $50 \%$ of a parenteral dose of cycloserine is excreted unchanged in the urine in the first 12 hours; a total of $65 \%$ is recoverable in the active form over a period of 72 hours. Very little of the antibiotic is metabolized. The drug may accumulate to toxic concentrations in patients with renal insufficiency; it may be removed from the circulation by dialysis. Cycloserine is also available for oral administration. The usual dose for adults is 250 to $500 \mathrm{mg}$ twice daily [5].

Reactions to cycloserine most commonly involve the central nervous system. Symptoms tend to appear within the first 2 weeks of therapy and usually disappear when the drug is withdrawn. Among the central manifestations are somnolence, headache, tremor, dysarthria, vertigo, confusion, nervousness, irritability, psychotic states with suicidal tendencies, paranoid reactions, catatonic and depressed reactions, twitching, ankle clonus, hyperreflexia, visual disturbances, paresis, and tonic-clonic or absence seizures [7, 8, 9]. Large doses of cycloserine or the concomitant ingestion of alcohol increases the risk of seizures. Cycloserinehas been found to induce positive symptoms in chronic schizophrenia and other studies have reported delirium associated with cycloserine $[10,11]$.

Cycloserine is contraindicated in individuals with a history of epilepsy and should be used with caution in individuals with a history of depression, as suicide is a risk [2, 12, 14]. The overall clinical scenerioin the patient forms this case report.

\section{Aim}

The aim of this report was to highlight a case of cycloserine induced-psychosis in a 22-year old male patient on treatment for drug resistant tuberculosis at the AluuMDRTBcentre of UPTH and to point to the reversible nature of the symptoms.

\section{Methodology}

The case report of the patient was retrieved and reviewed together with relevant literatures.

\section{The Case Report}

A 22-year old male patient who was diagnosed of tuberculosis in December, 2013 at a secondary health care facility and rediagnosed as multidrug resistant tuberculosis about 6 months ago. He had earlier being on anti-tuberculosis treatment-4 (ATT-4) (HRZE: Isoniazid, rifampicin, pyrazinamide, ethambutol) for 3 months, followed by ATT-2 (HR) regularly for 9 months, when he reported multiple swellings around his neck and groin. On fine needle aspiration cytology, these enlarged lymph nodes showed tubercular abscess. Development of abscess despite being on regular ATT for 12 months was considered as multidrug resistant tuberculosis (MDR-TB); He was later brought as a referral to the MDRTBcentre at Aluu and he was admitted into the facility and commenced on a course of Category IV antiTB regimen and hence, started on second line ATT, that is, cycloserine $750 \mathrm{mg}$ /day, ethionamide 500/day, ofloxacin 800/day in two divided doses and streptomycin $750 \mathrm{mg}$ per day along with continuation of ATT-2. After 1 week of commencing the antituberculosis regimen, patient began to manifest a range of unusual abnormal behaviours characterized by irrational talks, excessive talkativeness, irritable-aggressive behavior, poor sleep, refusal to eat, seeing strange images unseen by others in clear consciousness, restlessness, irritability, accusatory believes, occasional euphoria and unusual claims.

He was not a known psychiatric patients has not had any psychiatry consultation prior to this time. Apart from the illness that has interrupted his academic programme, there were no other stressors from school. Both parents are alive and well. The siblings are equally well adjusted. There was no history of use of psychoactive substances and there was no past or family history of any significant psychiatric or medical illness. His body weight as at the time of the report was $47 \mathrm{~kg}$.

Routine and other investigations including full blood count with differentials, electrolyte, urea and creatinine and urinalysis, thyroid function test done were all within normal ranges. Retroviral screening test was seronegative.

A consultation was made to the consultant neuropsychiatrist who came and reviewed. Mental state examination revealed a young man fairly kempt, uncooperative, good eye contact, he had increased psychomotor activity, irritable affect, but there was nil abnormal motor movement. Speech was incoherent, mood, he said; "I am not fine". Speech was irritable, there was visual hallucinations, paranoid and grandiose delusions. Cognition was fair and insight was lacking. Physical examination revealed mild palor, weight loss, with generalized lymphadinopathy. Brief Psychatric Rating Score (BPRS) [15] score was 62. High index of suspicion with score of 6 on Naranjo algorithm [16] (Naranjo et al., 1981) suggest probability of any of the potential drugs causing the psychosis. Since the patient was on several drugs with potential to cause psychotic reaction, we considered a provisional diagnosis of drug-induced acute psychosis.

Cycloserine was considered the most possible offending agent and the psychiatrist recommended that it be 
discontinued, whereas other drugs were continued under close observation., and the patient was placed on low dose benzodiazepine, $2.5 \mathrm{mg}$ nocte of nitrazepam, and vitamine $\mathrm{B}$ complex ii b. d. Psychoeducation was also done for the family members. Within 48hours, symptoms began to resolve. BPRS was 33 and within 72 hours, there was marked improvement particularly in the psychotic symptoms. Antipsychotic drugs were not commenced to allow for a brief period of watchful waiting and also to confirm the suspicion. At this point, a diagnosis of possible cycloserine-induced psychosis was entertained. We have obtained written informed consent to publish this case report.

\section{Discussion}

Cycloserine is a broad-spectrum antibiotic produced by Streptococcus orchidaceus. It was first isolated from a fermentation brew in 1995 and was later synthesized. Currently, cycloserineis used in conjunction with other tuberclostatic drugs in the treatment of pulmonary or extrapulmonary tuberculosis when primary agents (isoniazid, rifampin, ethambutol, pyrazinamide, streptomycin) have failed.4 Resistance was the reason for commencing the patient on cycloserine [4].

The incidence of depression, anxiety, and psychosis during MDR-TB treatment is found to be 13.3, 12.0, and $12.0 \%$, respectively [4]. But, specific role of cycloserine is not known, as the drug is almost always being used in combination in situations of MDR-TB. In this case, we stopped cycloserine only and continued all other drugs on development of psychosis, to isolate the switching effect of cycloserine from combinational effects. As expected with induced switch, the manic symptoms resolved quickly within 3 days.

The possible neurobiological mechanisms of induction of bycycloserine may be due to binding and modulation of NMDAR antagonists and partial agonists at the NMDAR-associated glycine (GLY) site by cycloserine for the antidepressant action [17] on dose of $500 \mathrm{mg}$ /day or above, [18] which may lead to manic switch in susceptible individuals. The role of NMDAR and glutamate-modulating agents such as D-cycloserine, riluzole, ketamine, and memantine is now being explored in resistant depression, mood disorders, and obsessive compulsive disorders [19, 20, 21]. Although there are reports [4] of depression with MDR-TB treatment, which may be the effect of coprescribed drugs in regime; but it cannot be attributed specifically to cycloserine, in view of its mechanism of action of NMDAR antagonism and partial agonism at the NMDAR-associated glycine.

This case supports the glutamatergic role of affective disorder and elaborates that patients being treated with cycloserine can develop psychosis which improves quickly within few days by stopping cycloserine with or without prescribing antipsychotic drugs. This implicates that adding cycloserine in psychotic, manic, or bipolar patients suffering from tuberculosis impose a risk of either manic switch or exacerbation of psychosis; whereas there are no reports about effects of cycloserine in patients of major depression and obsessive compulsive disorder, where some beneficial effects may be expected.

The patient was also on other drugs like pyrazinamide, isoniazid and ethambutol. Cycloserine is inhibitory for $\mathrm{M}$. tuberculosis in concentrations of 5 to $20 \mu \mathrm{g} / \mathrm{ml}$ in vitro. There is no cross-resistance between cycloserine and other tuberculosis agents. When given orally, as was the route of administration for the patient, $70 \%$ to $90 \%$ of cycloserine is rapidly absorbed. Peak concentrations 3 to 4 hours after a single dose. Cycloserine is distributed throughout body fluids and tissues.

There is no appreciable blood brain barrier to the drug and CSF concentrations are approximately the same as those in plasma. About $50 \%$ of a paranteral dose of cycloserineis excreted unchanged in the urine in the first 12 hours. Very little of the antibiotic is metabolized. These favourable pharmacokinetic properties may have been responsible for the rapid effects and subsequent manifestations of side effects of cycloserine in this patient $[4,7,8,9]$. The drug may accumulate to toxic concentrations in patients with renal insufficiency, however, there was no indication of obvious renal impairment from the investigations done in this patient.

Cycloserine is used for retreatment or when microorganisms are resistant to other drugs and must be given together with other effective agents, these conditions were all satisfied in this patient. The usual dose for adults is 250 to 500 $\mathrm{mg}$ twice daily, and 750mg dly was the dose for this patient.

Reactions to cycloserine most commonly involve the central nervous system. Symptoms tend to appear within the first 2 weeks of therapy and usually disappear when the drug is withdrawn. This was consistent with the patient as he started showing manifestation of side effects of cycloserine within days of its commencement [7, 8, 9]. The patient equally showed a good number of CNS manifestations. Among them were confusion, irritability, psychotic states with suicidal tendencies including visual hallucinations, and paranoid reactions, catatonic and depressed reactions $[4,7,8,9]$. Others still include twitching, ankle clonus, hyperreflexia, visual disturbances, paresis and tonic-clonic or absence seizures. However, these were not manifested by the patient.

Large doses of cycloserine or the concomitant ingestion of alcohol increases the risk of seizures, however, the patient does not take alcohol. The patient being reviewed had irrational talks, poor sleep and appetite, visual hallucinations, restlessness, irritability, paranoid and grandiose delusions, occasional euphoria $[7,8,9]$. The timing of the appearance of psychiatric symptoms in this patient- within the first 2 weeks, the fact that this patient had been on these other drugs for the past months, support the facts that cycloserine causes the psychiatric symptoms found in this patients. Except the on going medical condition, attempts were made to rule out other etiological factors. Ultimately, that the psychiatric symptoms substantially improved on withdrawing the suspected offending drug, cycloserine, all provide strong indications that this medication may have been responsible [5]. 


\section{Conclusion}

Our case highlights the importance of awareness regarding psychiatric adverse events of antitubercular agents and the reversible nature of the adverse events on drug withdrawal. We also suggest that caution should be exercised while administering cycloserine in MDR-TB patients because of a potential for causing psychiatric adverse events. This will help to prevent precipitation of adverse psychiatric symptoms and guarantee patients' optimal care.

\section{References}

[1] S. S. Shin, A. D. Pasechnikov, I. Y. Gelmanova, G. G. Peremitin, A. K. Strelis, S. Mishustin, Adverse reactions among patients being treated for MDR-TB in Tomsk, Russia, Int. J. Tuberc. Lung Dis. 11 (2007) 1314-1320.

[2] D. Yee, C. Valiquette, M. Pelletier, I. Parisien, I. Rocher, D. Menzies, Incidence of serious side effects from first-line antituberculosis drugs among patients treated for active tuberculosis, Am. J. Respir. Crit. Care Med. 167 (2003) $1472-1477$.

[3] L. P. Ormerod, N. Horsfield, Frequency and type of reactions to antituberculosis drugs: observations in routine treatment, Tuber Lung Dis. 77 (1996) 37-42.

[4] Vega P, Sweetland A, Acha J, Castillo H, Guerra D, Smith Fawzi MC, et al. Psychiatric issues in the management of patients with multidrug-resistant tuberculosis. Int $\mathrm{J}$ Tuberc Lung Dis. 2004; 8: 749-59.

[5] Coyne KM, Pozniaka AL, Lamordeb M \& Boffito M 2009. 'Pharmacology of second-line antituberculosis drugs and potential for interactions with antiretroviral agents'. AIDS, vol. 23, no. 4, pp. 437-446.

[6] E. Rouaud, J. M. Billard, D-Cycloserine facilitates synaptic plasticity but impairs glutamatergic neurotransmission in rat hippocampal slices, Br. J. Pharmacol. 140 (2003) 1051-1056.

[7] T. Torun, G. Gungor, I. Ozmen, Side effects associated with the treatment of multidrug-resistant tuberculosis, Int. J. Tuberc. Lung Dis. 9 (2005) 1373-1377.

[8] P. Baghaei, P. Tabarsi, D. Dorriz, M. Marjani, M. Shamaei, M. V. Pooramiri, Adverse effects of multidrug-resistant tuberculosis treatment with a standardized regimen: a report from Iran, Am. J. Ther. 18 (2011) 29-34.

[9] Sarkar S, Sood M. A patient of multidrug-resistant tuberculosis on category IV treatment regimen presenting with psychosis. Natl Med J India. 2011; 24: 244-5.
[10] J. R. Maldonado, Pathoetiological model of delirium: a comprehensive understanding of the neurobiology of delirium and an evidence-based approach to prevention and treatment, Crit. Care Clin. 24 (2008) 789-856.

[11] M. L. Gunther, A. Morandi, E. W. Ely, Pathophysiology of deliriumin the intensive care unit, Crit. Care Clin. 24 (2008) $45-65$.

[12] A. Pachi, D. Bratis, G. Moussas, A. Tselebis, Psychiatricmorbidity and other factors affecting treatment adherence in pulmonary tuberculosis patients, Tuberc. Res. Treat. Epub2013, Apr 15.

[13] A. Sharma, S. Malhotra, S. Grover, S. K. Jindal, Incidence, prevalence, risk factor and outcome of delirium in intensive care unit: a study fromIndia, Gen. Hosp. Psychiatry 34 (2012) 639-646.

[14] Arbex MA, Varella, M. C. L, SiqueiraHR, Mello, F. A. F 2010. 'Antituberculosis drugs: Drug interactions, adverse effects, and use in special situations. Part 2: Second-line drugs'. Brasilian Respiratory journal, vol. 36, no. 5, pp. 641-656.

[15] Overall JE, Gorham DR. The brief psychiatric rating scale. Psychol Rep. 1962; 10: 799-812.

[16] Naranjo CA, Busto U, Sellers EM, Sandor P, Ruiz I, Roberts EA, et al. A method for estimating the probability of adverse drug reactions. Clin Pharmacol Ther. 1981; 30: 239-45.

[17] Emmett MR, Mick SJ, Cler JA, Rao TS, Iyengar S, Wood PL. Actions of D-cycloserine at the N-methyl-Daspartate-associated glycine receptor site in vivo. Neuropharmacology. 1991; 30: 1167-71.

[18] Van BerckelBN, Lipsch C, Gispen-de Wied C, Wynne HJ, Blankenstein MA, van ReeJM, et al. The partial NMDA agonist D-cycloserine stimulates LH secretion in healthy volunteers. Psychopharmacology (Berl) 1998; 138: 190-7.

[19] Wilhelm S, Buhlmann U, Tolin DF, Meunier SA, Pearlson GD, Reese HE, et al. Augmentation of behavior therapy with D-cycloserine for obsessive-compulsive disorder. Am J Psychiatry. 2008; 165: 335-41.

[20] Sanacora G, Zarate CA, Krystal JH, Manji HK. Targeting the glutamatergic system to develop novel, improved therapeutics for mood disorders. Nat Rev Drug Discov. 2008; 7: 426-37.

[21] Stewart SE, Jenike EA, Hezel DM, Stack DE, Dodman NH, Shuster L, et al. A single-blinded case-control study of memantine in severe obsessive-compulsive disorder. J Clin Psychopharmacol. 2010; 30: 34-9. 